\title{
2000 中国大地坐标系
}

\section{杨元喜}

西安测绘研究所, 西安 710054

E-mail: yuanxi_yang@163.com; yuanxi@pub.xaonline.com

2008-12-19 收稿, 2009-04-07 接受

国家自然科学基金(批准号: 40774001, 40841021)和国家高技术研究发展计划(编号: 2007AA12Z331)资助项目

摘要 总结了构建 2000 中国大地坐标系及坐标框架的基本策略, 包括坐标系的定义, 坐标框架 关键词 建设层次, 实现 2000 中国大地坐标框架的函数模型、随机模型以及平差方法的改进. 首先利用 国际高精度 IGS 站与我国连续运行 GPS 网联合解算, 构成我国 2000 坐标系的基本骨架，保证我 国 2000 坐标系与国际高精度地心坐标系统一致; 在高精度 IGS 站坐标控制下, 将我国已经建成 的 6 个全国范围 GPS 大地控制网进行优化集成，建立了具有 2500 多点的高精度的、统一的 2000 国家 GPS 大地控制网” (历元为 2000.0), 形成了 2000 中国大地坐标系的基本框架; 再将全国天 坐标系统 坐标框架 大地控制网 GPS 控制网 基准 文大地网与 2000 国家 GPS 大地控制网进行联合平差, 进一步加密了我国大地坐标系框架, 加强 了我国天文大地网的现势性, 提高了天文大地网的精度, 统一了国家大地测量基准. 通过具有针 对性的数据融合方法处理后的中国大地坐标框架, 消除了各类大地网的基准差, 减弱了系统误 差和异常误差的影响. 最后对启用 2000 中国大地坐标系的意义以及现有中国大地坐标框架存在 的问题进行了描述.

\section{1 我国大地坐标系现状}

地球坐标系统(TRS)的建设包括坐标系定义和地 球参考框架建设. 坐标系的定义包括: 坐标系原点、 轴向和尺度; 一个地球参考系必须由精确测定其坐 标和速度的物理点集实现, 这些点集称为地球参考 架(TRF).

由于历史和技术的原因，我国在不同时期曾建 立和使用过多种大地坐标系(1954 年北京坐标系、新 1954 年北京坐标系、西安 1980 坐标系、地心 I 号 (DX- I )和地心 II 号(DX- II)坐标系、WGS-84, 这些 坐标系为国民经济建设、航空、航天、航海及科学研 究提供了重要支撑. 但是多种坐标系共存也给实际 应用带来了许多实际问题。一是容易造成地理空间 信息的混乱; 二是目前使用最为广泛的新旧 1954 年 北京坐标系和西安 1980 坐标系属于局部参心坐标系, 精度偏低，难以满足航空、航天、航海及国防建设的
需要; 三是曾经使用过的DX-I 和DX- II坐标系, 只 是二套相对新 1954 年北京坐标系的坐标转换参数, 没有实际的坐标框架, 坐标转换参数的精度较低, 难 以满足科学研究的需要 ${ }^{[1,2]}$. 为此, 多位学者提出建 立我国新一代地心坐标系的设想 ${ }^{[2 \sim 4]}$.

（i ） 1954 年北京坐标系. 1954 年北京坐标系是 通过我国东北呼玛、吉拉林、东宁 3 个基线网与前苏 联远东大地控制网相联接, 将前苏联 1942 年普尔科 沃(Pulkovo)坐标系延伸至我国的一个坐标系. 于是, 1954 年北京坐标系实际上是前苏联 1942 年普尔科沃 坐标系在我国的扩展和延伸.

1954 年北京坐标系建立后, 我国天文大地网采 取边布设边平差的方式，获得了约 40 千点的坐标， 从而构成了 1954 年北京坐标系的基本参考框架. 1954 年北京坐标系存在以下问题: (1) 采用的克拉索 夫斯基椭球与现代椭球相比，长半轴大了 $108 \mathrm{~m}$, 扁 
率倒数大了 $0.04 ;$ （2) 椭球定位定向有较大偏差, 与 我国大地水准面存在着自西向东明显的系统性倾斜, 最大倾斜量达 $65 \mathrm{~m}$; 椭球短轴的定向也不明确; (3) 坐标系原点不在北京, 而在前苏联的普尔科沃, 取名 为“北京坐标系”名不符实; (4) 几何大地测量与物理 大地测量采用的椭球也不统一, 给实际使用带来不 便; (5) 坐标精度偏低, 相对精度为 $5 \times 10^{-6}$ 左右; (6) 由于采用了分区局部平差法, 系统误差累积明显, 导致大地网产生扭曲和变形，区与区之间产生裂隙; （7）较低精度的二维大地测量成果与高精度的三维 卫星大地测量成果不相匹配, 引起使用上的麻烦.

(ii ) 1980 年西安坐标系. 1980 年西安坐标系是 经全国天文大地网整体平差而建立的另一个参心坐 标系, 采用的参考椭球比较合适, 坐标轴的指向明确, 参考椭球面与我国大地水准面吻合较好, 椭球定位 比较符合中国的实际. 大地原点位于陕西泾阳县永 乐镇.

1980 年西安坐标系建立后, 进行了全国天文大 地网整体平差. 通过平差消除了分区局部平差和逐 级控制造成的影响, 提高了平差结果的精度. 平差后 获得约 50 千点的坐标, 构成了 1980 年西安坐标系的 基本参考框架. 但全国天文大地网整体平差时, 采用 了大地原点固定的、以天文方位角与起始边作控制的 单点自由网平差法, 天文方位角与起始边的系统误 差无条件地带入整网平差结果中, 整体平差后的全 国天文大地网存在较大的系统性扭偏.

1980 年西安坐标系仍然存在以下问题: 一是只 能提供二维坐标, 不能提供高精度三维坐标; 二是采 用了国际大地测量协会(IAG)1975 年推荐的椭球, 该 椭球与 IERS 推荐的椭球相比, 长半轴大了 $3 \mathrm{~m}$, 这可 能引起约 $5 \times 10^{-7}$ 量级的长度误差; 三是椭球短轴指 向 JYD1968.0 极原点, 与国际上通用的椭球短轴指向 不一致; 四是椭球定位没有顾及到占中国全部国土 面积近三分之一的海域范围. 尽管 1980 年西安坐标 系比 1954 年北京坐标系有所改善, 但并没有发生实 质性的变化.

同时，考虑到不影响已有海量基本比例尺地形 图的使用, 通过对 1980 年西安坐标系进行平移转换, 又建立了新 1954 年北京坐标系(整体平差转换值). 该坐标系采用了与 1954 年北京坐标系一样的克拉索 夫斯基椭球, 精度与 1980 年西安坐标系基本一致.

(iii) DX- I 和 DX- II坐标系. 为了适合航天技
术的发展, 20 世纪 70 年代和 80 年代, 我国先后建立 了两套地心坐标系统, “DX- I”和“DX- II”. 这两套 地心坐标系实际上只求解了两套坐标转换参数. “DX-I ”只有 3 个平移参数而不包含旋转参数和尺度 变化参数, 仅表示 1954 年北京坐标系椭球中心相对 于地球质量中心的位置, 忽略了坐标轴向的不平行, 所用的椭球大小也不一样. 此外, DX-I 所使用的资 料也有限、原始数据不精确、处理方法也不够完善. 因此, 由“DX-I”转换参数转换的坐标精度仅在 $15 \mathrm{~m}$ 左右. 20 世纪 80 年代, 我国布设了全国多普勒网(37 个点), 1982 年又布测了卫星动力测地网 (7 个点), 1985 年完成了定位解算, 获得了全国范围相应点的 地心坐标. 同时, 完成了全国天文大地网整体平差, 确定了约 50 千点的大地坐标, 并求得了新的地心坐 标转换参数, 取名为“DX- II”. “DX- II”由 3 个平移参 数、 3 个旋转参数和 1 个尺度参数组成, 各坐标分量 转换精度约在 $5 \mathrm{~m}$ 左右.

\section{2 国际主要国家大地坐标系统建设概况}

\section{1 北美坐标系发展概况}

( i ) NAD83 坐标系. 1986 年北美大陆(美国、 加拿大、墨西哥)通过地面控制网和空间测量网(多普 勒数据、VLBI 数据)联合平差, 建立了 NAD83 地心 坐标系. 联合平差的大地控制网由 250 千点组成. 多 普勒数据和 VLBI 数据用来确定 NAD83 参考框架的 定向, 最初的定义要求 NAD83 参考框架与 WGS-84 的轴向一致、原点重合. 但与 WGS-84 不同的是, NAD83 采用 GRS80 参考椭球，参考椭球的原点与地 球质心重合.

北美 NAD83 第 2 版 NAD83 增加了 GPS, VLBI 和 SLR 观测信息. 统一处理后 NAD83 地心坐标系与 地球质心的重合度大约为 $2 \mathrm{~m}$, 空间坐标轴定向为 0.03 ", 网的尺度达到 $0.0871 \times 10^{-6}$, 大地高的精度提 高到 0.6 m. 1994 年出台了第 3 版 NAD83, 它考虑到 了 1989 年国际地球参考框架(ITRF89)的观测成果.

(ii ) WGS-84 坐标系. GPS使用的世界大地坐 标系是WGS-84, 它的定义与国际地球参考系(ITRS) 一致 ${ }^{[5,6]}$. WGS-84 由 12 个地面跟踪站和卫星星历共 同维持. 经过 1994 年和 1996 年两次精化, WGS-84 与ITRF符合精度(RMS)在 $5 \mathrm{~cm}$ 以内; 经过 2001 年的 再次精化, WGS-84 坐标系与ITRF2000 每一坐标分量 的符合精度已达 $1 \mathrm{~cm}$. 


\section{2 中、南美洲坐标系发展概况}

1995 年南美洲 11 个国家开展了近 58 个点的 GPS 会战(即 SIRGAS 计划), 建立了与 ITRS 相一致的地 心参考系 SIRGAS. SIRGAS 与 ITRF94 在历元 1995.4 一致. 坐标外部符合精度 $(\mathrm{RMS})$ 为 $3 \mathrm{~cm}$; 坐标分量的 内部精度(中误差)为 $4 \mathrm{~mm}$. 南美许多国家在 SIRGAS 的基础上, 也建立了各自的地心参考框架, 地心坐标 内部精度约为 $2 \mathrm{~cm}$.

\section{3 欧洲坐标系发展概况}

欧洲参考系(ETRS)固联于欧洲板块的稳定部分. 相应的欧洲参考框架(EUREF)分为 3 级 ${ }^{[7]}$ : A 级相对于 ITRF89 框架的精度达 $1 \mathrm{~cm}$, B级在给定历元的坐标精 度达 $1 \mathrm{~cm}$, C级相对于ITRF89 的精度达 $5 \mathrm{~cm}$. 欧洲参 考框架是ITRF 在欧洲的加密, 不仅坐标精度高, 而 且坐标维持手段完善. 现在欧洲已有 90 多个GPS永 久跟踪站维持EUREF.

\section{4 俄罗斯坐标系发展概况}

从 20 世纪 70 年代起前苏联决定改用全球地心坐 标系. 1988 年俄国斯军方开始实施新的统一地心坐 标系CK-90，并规定CK-90 与CK-42 并用. 在民用方 面, 俄国斯从 1995 年起改用CK-95 新系统. 之后俄国 斯国防部又推出了更精确的地心坐标系PZ-90, 精度 为 $1 \sim 2 \mathrm{~m}$, 控制点的相对精度为 $0.2 \sim 0.3 \mathrm{~m}^{[8]} .2007$ 年 统一采用国家地心坐标系PZ-90.

\section{5 邻近国家坐标系发展概况}

邻近国家大地测量控制网和坐标系统的建设近 来也都取得了长足进展 ${ }^{[6]}$. 日本大地基准JGD2000 采 用ITRS的定义, 历元为 1997.0, 由 1200 个 GPS连续运 行站(GEONET)协同 64000 个一等、二等、三等经典 大地点维持. 蒙古建立了新的国家大地坐标框架 MON-REF97, 该框架和WGS-84 保持一致. 新西兰于 1998 年建立了新的三维地心坐标系NZGD2000, 基准 与ITRS一致, 参考历元为 2000.01.01. 澳大利亚地心 基准GDA1994.0, 依赖于 78 个GPS点组成的澳大利 亚国家控制网ANN和永久GPS跟踪网ARGN维持，对 应于ITRF92 坐标(历元 1994.0), 坐标精度约几个厘米. 韩国和马来西亚分别推出国家三维地心坐标系统 KGD2000 和NGRF2000. 这两个坐标系均以ITRF97 为参照, 历元同为 2000.0 .

\section{0 中国大地坐标系建设现状}

2000 中国大地坐标系(简写为CGCS2000)的定义 与ITRS的协议一致, 即坐标系原点为包括海洋和大 气的整个地球地质量中心; 定向的初始值由 1984.0 时BIH定向给定，而定向的时间演化保证相对地壳不 产生残余的全球旋转 [4]; 长度单位为引力相对论意义 下的局部地球框架中的米. CGCS2000 的参考历元为 2000.0 .

我国地心坐标系所采用的参考椭球的定义常数 为 $a$ (赤道半径)、 $f$ (椭球扁率)、 $G M$ (地心引力常数) 和 $\omega$ (地球自转速度). 上述 4 个参数值中, $a, f, \omega$ 值与 1980 参考椭球(GRS1980)一致, GM值与WGS-84 的椭 球参数值一致 ${ }^{[4]}$. 应该指出, 由于 GPS实时定位采用 的是WGS-84 坐标系, 该坐标系与CGCS2000 的椭球 扁率f值有微小差异, 在赤道上只引起 $1 \mathrm{~mm}$ 误差, 可 以认为GPS实时定位结果也属于CGCS2000 坐标系成 果.

2000 中国大地坐标系的实现分 3 个层次(见图 1).

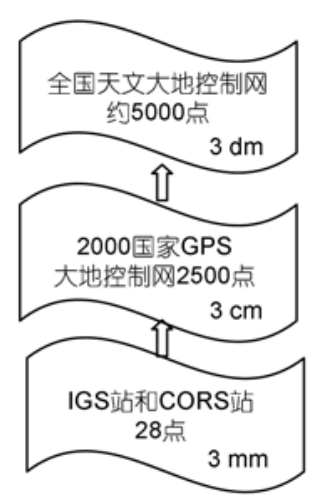

图 12000 国家大地坐标框架层次

第一层次为 CGCS2000 连续运行 GPS 网. 我国 维持 CGCS2000 主要依靠连续运行 GPS 观测站, 它 们是 GPS2000的骨架, 其坐标精度为毫米级, 速度精 度为 $1 \mathrm{~mm} / \mathrm{a}$.

第二层次为“2000 国家GPS大地控制网”[9 11]，包 括中国全部领土和领海的高精度GPS网点, 即全国 GPS一、二级网 ${ }^{[12]}$ 、国家GPS A, B级网 ${ }^{[13]}$ 、地壳运动 监测网和地壳运动观测网络工程网 [14]，共约 2500 多点, 它是在国际 IGS 站以及中国地壳运动观 测网络工程网点的控制下经联合平差组成. 其三维 地心坐标精度约为 $3 \mathrm{~cm}$.

第三层次为全国天文大地控制网(约有 50 千点). 
它是CGCS2000 的加密框架. 它由全国天文大地网与 2000GPS控制网联合平差后的网点坐标体现, 三维点 位误差约为 $0.3 \mathrm{~m}^{[15]}$, 大地高误差不超过 $0.5 \mathrm{~m}$.

\section{0 中国大地坐标框架建设主要策略}

\section{1 第一层次坐标框架一连续运行参考站网}

为使我国 2000 坐标系与 ITRS 尽量一致, 我们选 择了国际 IGS 核心站控制我国 GPS 连续运行参考站 的数据处理. 先对 IGS 核心站进行初选和统计检验, 初选原则是: (1) IGS 站的站址尽量全球均匀分布; (2) 具有可靠的、高精度的 ITRF97 的坐标和速度; (3) 我国地壳运动观测网络工程网运行期间这些 IGS 站具有良好的观测质量. 如此, 我们选定了 47 个 IGS 站作为控制框架, 对网络工程基准网的单日解进行 松驰约束平差, 利用已求得的速度进行历元归算, 得 到一个无基准的整体解; 然后利用 IERS 公布的各 ITRF 相对于 ITRF97 的 7 个转换参数 ( 3 个平移、 3 个 旋转、 1 个尺度), 将连续运行参考站坐标转换为 ITRF97 坐标, 平差后站坐标精度约为 $3 \mathrm{~mm}$.

\section{2 第二层次坐标框架 -2000 国家 GPS 大地控 制网}

2000 国家 GPS 大地网是多个 GPS 网的集成, 覆 盖了我国整个大陆及部分沿海岛屿. 2000 国家 GPS 网(简称 2000 网) 所采用的观测数据跨度为 $12 \mathrm{a}$, 即从 1988 年到 2000 年. 由于测区地理环境和气候条件差 异悬殊，野外作业单位不同、采用的仪器类型不同， 实测方案、网形结构等都不尽相同, 所以各子网存在 明显的局部系统误差和异常误差，而且精度分布不 均. 为了获得高精度统一的框架点坐标, 采用了如下 技术设施:

( i ) 平差基准的统一. 为了减弱整体数据处理 时的精度损失, 2000 网整体平差时, 选择ITRF97 坐 标框架为基准, 参考历元为 2000.0, 以全球高精度 IGS站坐标作为控制. 将各历元GPS观测量归算到同 一参考框架、同一参考历元 ${ }^{[9]}$.

(ii) 子网之间的系统误差补偿. 为了减弱各子 网存在的系统误差(包括基准系统差、观测系统差、仪 器系统差、轨道和星历系统差、地壳形变系统差等), 在全网整体平差中, 以IGS站和连续运行GPS点为框 架, 对各子网基线向量分别引入尺度参数 $m$ 和旋转参 数 $\left(\omega_{x}, \omega_{y}, \omega_{z}\right)$. 应用具有系统误差参数的平差模型, 一 方面保证了各子网平差基准的一致性，同时也削弱了
各子网间的系统误差及地壳形变的影响 $[9,10,16]$.

(iii) 异常误差影响控制. 2000GPS网整体平差 前, 每个同步观测区均进行了异常误差探测. 若标准 化残差大于 3 , 则该观测为可疑异常观测, 相应的同 步观测区暂时不参加平差计算, 以便为后续抗差估 计迭代计算提供较可靠的参数初值. 此后, 应用双因 子相关观测抗差估计理论进行参数估计 ${ }^{[17 ~ 19]}$, 如此, 既能控制相关异常误差影响, 又能保证观测向量的 等价权矩阵的对称性和原有内在相关性不变, 且计 算相对简单, 该方案等价于基于方差膨胀模型的抗 差估计法 ${ }^{[20]}$ ，但计算相对简单.

(iv) 同步观测区及各子网随机模型误差补偿. 各子网标称精度往往过于乐观, 存在随机模型误差. 为了控制随机模型误差对整体GPS网平差结果的影 响, 在处理各同步区子网平差和最后的整体网平差 中, 均应用方差分量估计重新估计各子网观测量的 精度和权. 为了控制个别异常误差对方差分量及参 数估计值的影响, 采用了新的抗差方差分量估计法 [21].

2000 国家 GPS 大地控制网的建成，统一了我国 大地测量坐标框架, 统一后的 GPS 网的精度有了明 显提高, 平均点位坐标精度优于 $3 \mathrm{~cm}$.

\section{3 第三层次坐标框架一一全国天文大地网}

经过我国大地测量工作者 70 多年努力而建成的 全国天文大地网具有较高的相对精度和密度, 是国 家大地测量的宝贵财富. 为了加密我国大地坐标框 架, 我国从 1991 年起开始启动“全国天文大地网与空 间大地控制网的联合平差工程”(简称联合平差工程). 参加联合平差的点近 50 千个, 平差观测量 440 千余 条, 未知数约为 180 千个.

(i ) 天文大地网平差的基准. 为使新的联合平 差点位坐标统一到CGCS2000, 联合平差时将空间大 地控制网平差结果施加 $1 \sigma$ 约束. 理论上, 对参考点 坐标施加 $1 \sigma$ 约束, 等价于天文大地网与 2000 网观 测数据进行了整体平差, 因而原有的坐标框架不变. 如此, 不仅保证了平差结果的基准与IGS站坐标基准 一致，而且控制了联合平差的误差转移 ${ }^{[22]}$.

(ii ) 数据归算. 为了数据归算的严密性, 天文 成果进行了极移改正、综合时号改正和人仪差改正 ${ }^{[23]}$; 其他地面观测成果进行了垂线偏差改正和高程异常 改正 ${ }^{[24,25]}$.

由于地面观测数据只是相对量, 且站间距离仅 


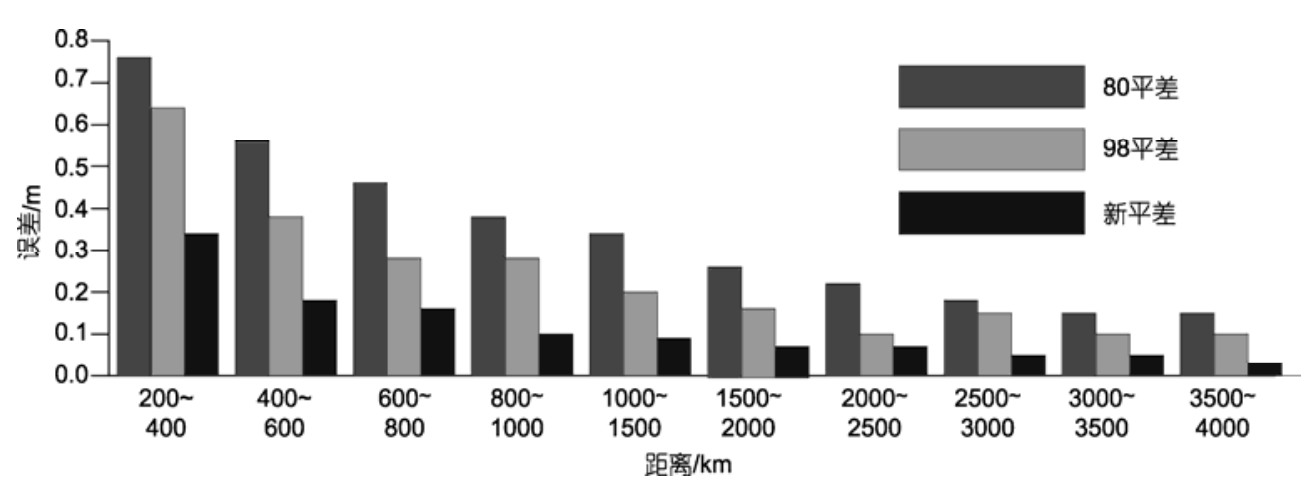

图 2 新的联合平差与 1980 和 1998 年联合平差相对精度比较

为 $10 \mathrm{~km}$ 左右, 在 GPS 网的强制控制下, 地面网不再 加地壳形变改正.

(iii) 模型改进和质量控制. 在函数模型方面, 对地面各类测边网分别引入 9 个尺度参数, 以顾及尺 度系统误差的影响; 在随机模型方面, 分别对垂直角 观测和水平方向观测进行了粗差探测和剔除 ${ }^{[26,27]}$, 采用方差分量估计重新标定了各类观测量的方差和 权，采用抗差估计控制了各类异常观测误差的影响， 从整体上加强了全国大地控制网的质量.

(iv) 法方程解算方案. 由于联合平差法方程矩 阵为稀疏矩阵, 利用Helmert分块法将全国范围的大 地网按南北纵线将天文大地网分成 16 块, 平均每块 约 3 千点, 将未知参数分为“内部”和“联系”两类参数, 使各块内部参数的解算变成相对独立过程且能并行 计算 ${ }^{[28]}$. 为减少联系未知数, 块与块之间的分界按边 连接，除分界线上的点外，各块之间没有观测量相联 系. 实际解算时, 每区单独解算, 消去区内未知数, 求出仅含联系未知数的约化方程. 全部分区解算完 成后, 把所有的分区约化方程及空间网点坐标权矩 阵相加, 建立全网的总约化法方程, 解出联系未知数, 然后回代求解各区内未知数, 计算各点位坐标, 并进 行平差检核. 区内解算采用了迭代约化代替求逆约 化, 利用联系末知数的逆矩阵和区内未知数的初步 逆矩阵, 计算出准确的区内法矩阵的逆矩阵, 实现对 区内点的精度估计. 总约化方程采用求逆解算, 并估
计了所有联系点的坐标精度.

经过外部检核, 联合平差后的三维点位坐标外 部符合精度(RMS)优于 $0.4 \mathrm{~m}$; 内部精度(中误差)优于 $0.15 \mathrm{~m}$; 新的联合平差结果的相对中误差仅为 1998 年联合平差结果的二分之一, 为 1980 年平差结果的 三分之一(见图 2). 显然联合平差工程的完成显著改 善了国家天文大地控制网的精度.

\section{0 国家大地坐标框架存在的主要问题}

(1) 2000 坐标框架虽然满足国民经济建设和国防 建设急需, 但该坐标框架的密度仍然不够, 尤其是西 部困难地区, 不仅控制点少, 而且观测精度也相对较 差, 很难满足国民经济建设和西部开发的需要.

(2) 2000 坐标框架广度不够, 广大海洋和岛礁几 乎没有控制, 很难满足航海安全、海洋开发和国防建 设的需要.

(3) 2000 坐标框架总体精度仍然偏低, 尚不能提 供点位的三维变化信息, 显然不能满足减灾防灾和 地球动力学研究的需要.

(4) 点位归算十分困难. 我国 2000 框架采用的 是 ITRF97 框架, 2000.0 历元, 这对于目前广泛采用的 GPS 精确定位(ITRF2005 框架和当前历元)带来不便, 若要进行转换, 必须有高分辨率的速度场资料, 以便 实施已知点从 2000 年至当前历元的点位归算. 显然 目前的条件尚不具备. 因此 2000 中国坐标框架仍应 该实时进行更新, 尤其是历元的更新.

致谢本论文是在多项大型大地测量工程基础上完成的, 包括“2000 国家 GPS 大地网数据处理工程”、“全国天文大地 网与空间网联合平差工程”、“全国 GPS 一 二级网测量工程”、“国家 GPS A, B 级网观测工程”、“地壳运动监测 网”和“地壳运动观测网络工程”等, 没有丰富的高精度观测数据的支持, 建立全国高精度地心坐标框架是不可能 的. 


\section{参考文献}

顾旦生，张莉，程鹏飞，等. 我国大地坐标系发展目标. 测绘通报, 2003, 3: $1-4$

魏子卿. 关于建立新一代地心坐标系的意见. 见: 地面网与空间网联合平差论文集 [三]. 北京: 解放军出版社, 1999. 85- 90

3 陈俊勇. 改善和更新我国大地坐标系统的思考. 见: 陈俊勇, 主编. 大地测量论文集一一祝贺陈永龄院士 90 寿辰. 北京: 测 绘出版社, 1999

4 魏子卿. 我国大地坐标系的换代问题. 武汉大学学报(信息科学版), 2003, 28: 138-143

5 陈俊勇. 关于中国采用地心 3 维坐标系统的探讨. 测绘学报, 2003, 32: 283-288

6 陈俊勇. 邻近国家大地基准的现代化. 测绘通报, 2003, 10: 1-3

7 Adam J, Augath W, Boucher C, et al. The European Reference System coming to age, International Association of Geodesy Symposia, Vol. 121. In: Schwarz, ed. Geodesy Beyond 2000—The Challenges of the First Decade. Berlin Heidelberg: Springer, 2000. 47一 54

段五杏, 王刚, 袁学伟, 等. GPS 二级网的数据处理. 见: 地面网与空间网联合平差论文集[三]. 北京: 解放军出版社, 1999. $137-148$

李艈麟，刘经南，葛茂荣，等.中国国家 A 级 GPS 网的数据处理和精度评估。测绘学报, 1996, 25: 81-86

王敏, 沈正康, 牛之俊, 等. 现今中国大地地壳运动与活动块体模型. 中国科学 D 辑: 地球科学, 2003, 33(增刊): 21一 32

Yang Y, Zha M, Song L, et al. Combined adjustment project of national astronomical geodetic networks and 2000' national GPS control network. Prog Nat Sci, 2005, 15: 435-441 [DOI]

16 施闯, 刘经南, 姚宜斌. 高精度 GPS 网数据处理中的系统误差分析. 武汉大学学报(信息科学版), 2002, 27: 148一-152

17 Yang Y X. Robust estimation for dependent observations. Manuscript Geod, 1994, 19: 10—17

18 Yang Y X, Song L J, Xu T H. Robust estimator for correlated observations based on bifactor equivalent weights. J Geod, 2002, 76: $353-358[\mathrm{DOI}]$

19 Yang Y X, Song L J, Xu T H. Robust parameter estimation for correlated geodetic observations. Acta Geod Cartogr Sin, 2002, 31: 18 $-24$

20 Liu J N, Yao Y B, Shi C. Theory research on robustified least squares estimator based on equivalent variance-covariance. Sci Surv Map, 2000, 25: 1-5

21 Yang Y X, Xu T H, Song L J. Robust estimation of variance components with application in Global Positioning System network adjustment. J Surv Eng, 2005, 131: 107-112[DOI]

杨元喜. 全国天文大地网与空间大地网联合平差二期工程总体方案. 见: 地面网与空间网联合平差论文集 [四]. 北京: 解放 军出版社, 2003. 66-71

张红英, 谈国良, 张新兵, 等. 地面网与空间网联合平差中天文成果的改算. 地面网与空间网联合平差论文集 [四]. 北京: 解 放军出版社, 2003. 211-220

孙风华, 陈春旺, 孔维兵, 等. 我国陆海 $1^{\prime} \times 1^{\prime}$ 平均重力异常数字模型的建立及可靠性检验. 地面网与空间网联合平差论文 集[四]. 北京: 解放军出版社, 2003.190-196

张传定, 吴晓平, 孙风华, 等. 中国 $1^{\prime} \times 1^{\prime}$ 数字大地水准面和垂线偏差模型的建立. 地面网与空间网联合平差论文集 [四]. 北 京: 解放军出版社, 2003. 155-158

欧吉坤. 粗差的拟准检定法(QUAD 法). 测绘学报, 1999, 28: 15-20

宋力杰, 杨元喜. 论粗差修正与粗差剔除. 测绘通报, 1999, 38: 5-6

宋力杰, 欧阳桂崇. 超大规模大地网分区平差快速解算方法. 测绘学报, 2003, 32: 204-207 